\title{
Improved Face Detection Algorithm in Mobile Environment
}

\author{
Sang-Burm Rhee and Yong-Hwan Lee \\ Dept. of Elect. \& Com. Eng., Dankook Univ., Korea \\ sang107@dku.edu
}

\begin{abstract}
In this paper we propose a new algorithm to be able to implement fast and accurate search for the characteristic points in a face by scanning with Mobile Camera Phone (MCP). The algorithm transforms the RGB color space to the YUV (i.e.,use a matrixed combination of Red, Green and Blue to reduce the amount of information in the signal.), and detects the face color reducing the influence of brightness by Min-max normalization and histogram equalization. Experimental results show that this algorithm has more accurately than previous method.
\end{abstract}

\section{Introduction}

Many researches to apply the face recognition to mobile systems such as mobile phones, and PDA have been proceeding in accordance with the mobile computing environment is rapidly advanced. The combination of the face recognition and the mobile system could be applicable to security checking for criminals and visual communication. We transmit an image from the mobile camera to a server using a PDA or a MCP, and then recognize the face by processing of the image transmitted [1] 2. The face recognition has been generally approached with the pattern recognition to use image halftoning or edge in a static image as its features. Kanade [3] 4] provided a method that automatically recognizes faces using static features including face components such as the contour, the eye, the nose and the mouth. This method resulted in reliable face recognition at pictures containing face. But it is useful exclusively for the curves to have few parameters, and it requires long computation time and a lot of calculations and spaces, and the accuracy of the detection depends on the size to quantize the parameter spaces.

\section{Authentication of the Face Entered with a MCP}

Skin Color Detection using YUV: RGB values of pixels in the image are transformed to YUV color spaces by equation(1) in order to detect a face from the image entered with a MCP, the illumination calibration with Min-Max Normalization is prerequisite during the pre-processing for the accurate face detection. Histogram equalization enhances the performance of the image which 
brightness is secund into the one direction can be used for the intensity equalization. After reducing the illumination impact by applying min-max normalization and histogram equalization to the brightness component in $\mathrm{Y}$ component, we transform YUV values to RGB form.

$$
\left[\begin{array}{l}
R \\
G \\
B
\end{array}\right]\left[\begin{array}{ccc}
1.164 & 1.596 & 0 \\
1.164 & -0.391 & 0.813 \\
1.164 & 0 & 2.018
\end{array}\right]\left[\begin{array}{c}
Y-16 \\
U-128 \\
V-128
\end{array}\right]
$$

The skin color is extracted by using the skin color model. It is the skin color extraction image at the image processed with the Min-max normalization. We defined light brightness as the optimized light of the skin color extraction in the skin color model. Defining both quite dark and bright images to the suitable light one by histogram equalization allows to extract the skin color regardless of light brightness.

Detection of Characteristic Points: For preventing errors from occurring when dark illumination is exposed or colors similar to pupil color are distributed, characteristic points in a face should be clearly expressed unlikely the skin color in order to find characteristic points such as the nose and the mouth in a face. we let the distance between both eyes $L$, and define $L^{\prime}$ for a distance to lower as much as $L$ from the middle position between two eyes, and then would see a lot of features for the nose at the $L^{\prime}$. The lip range is searched by using colors the lib has, by starting from $L^{\prime}$ with these feature.

Face Authentication using Support Vector Machine(SVM): SVM mainly distinguishes objects as two categories. Learning samples consist of $N$ objects, and let's express the vector $x_{i}$, comprising of $p$ variables for the $i$ th object, and $y_{i}$ for the category classified already, corresponding to the $x_{i}$. We assume that there are two categories for $y_{i}$, either +1 or -1 . We consider a separable hyperplane for the positioning $N$ objects, consisting of two categories, into a $p$-dimensional space. In this case, it is useful for the case that a hyperplane is not deterministic. let's consider two parallel hyperplanes as below.

$$
H_{1}: y=w^{\prime} x+b=1, H_{2}: y=w^{\prime} x+b=-1
$$

At this time, the hyperplanes $H_{1}$ and $H_{2}$ in equation(2) pass through the object nearest to the category +1 and category -1 respectively at the separating hyperplane. Therefore, the margin between $H_{1}$ and $H_{2}$ is $2 /\|w\|$. So, the optimization problem for this case can be expressed as follow.

$$
\operatorname{Max} \frac{2}{w^{\prime} w}\left(\operatorname{or} M i n \frac{w^{\prime} w}{2}\right) \text { subject to } y_{i}\left(w^{\prime} x_{i}+b\right) \geq 1
$$

Inducing Lagrange by introduction of non-negative Lagrange coefficient may cause the optimization problem, called Primal problem. Applying KKT condition into this problem with any object $i$ which $\alpha_{i}>0$, we can obtain the equation (4).In the optimal solution, object with $\alpha_{i}>0$ is support vector, placed on $H_{1}$ or $H_{2}$, otherwise, $\alpha_{i}=0$. 


$$
w=\sum_{i=1}^{N} \alpha_{i} y_{i} x_{i}, \quad b=\frac{1-y_{i} w^{\prime} x_{i}}{y_{i}}
$$

Face Authentication: In order to authenticate a face using SVM, data learning process is needed. we assigned +1 and -1 as reference values for the case of being matched between faces and not being matched respectively, by analyzing the face group in the database and the face in images entered. We normalized SVM learning data to $80 \times 80$ size, and entered the images created by quantization by 3 bits from the normalized images into the input images for SVM. In the SVM learning process, we assigned +1 and -1 as the reference values for the case of being matched between faces and not being matched respectively, letting SVM to be learned.

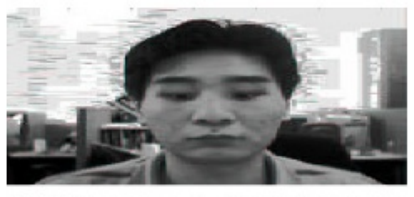

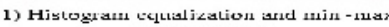
nommilization

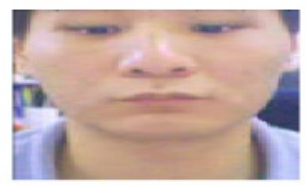

3) Dotection of face range

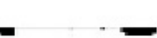

5) Detection of the eye range

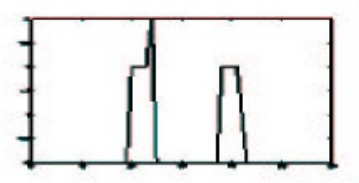

7) Detection of the nose range

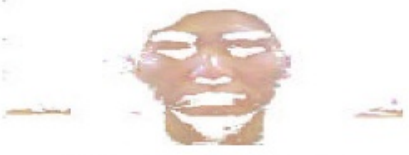

2) Derection of the skin col or

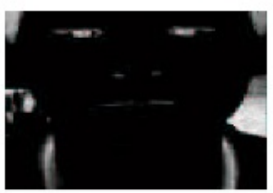

1) Tranaformation to $V$ '

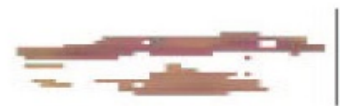

6) Detection of the lip range

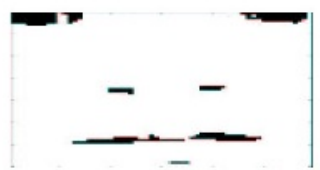

8) Detection of the characteristic pointis in as faces:

Fig. 1. An experimental process

Table 1. Comparison of searching time in a face

\begin{tabular}{|c|c|}
\hline & Time \\
\hline PCA & $1.8 \mathrm{sec}$ \\
\hline PCA+LDA & $2.3 \mathrm{sec}$ \\
\hline Proposed Method & $1.4 \mathrm{sec}$ \\
\hline
\end{tabular}


Table 2. Comparison of Authentication ratio

\begin{tabular}{|c|c|c|c|}
\hline & $\begin{array}{c}\text { The number of } \\
\text { total data }\end{array}$ & $\begin{array}{c}\text { The number of } \\
\text { Authenticated data }\end{array}$ & $\%$ \\
\hline PCA & 200 & 158 & $79 \%$ \\
\hline PCA+LDA & 200 & 176 & $88 \%$ \\
\hline Proposed method & 200 & 185 & $92 \%$ \\
\hline
\end{tabular}

\section{Experimental Environment and Result}

To estimate the proposed method, we assume a system model for the face detection. In experiment environment, we use machines with running Windows XP Professional operation system, SCH-V300 Handset and BizCardReader 600c as input device, and implement with MS Visual C++6.0 using QCIF $(320 \times 240)$ image size.

In Fig.1, we illustrated the experimental process for detecting the characteristic points in a face from the image entered with a MCP. Pre-processed with Min-max normalization and histogram equalization for the accurate detection given in 1). With this result, extracted the skin color(2)), and then detected the face range $(3))$. After this, we detected the eye range, the lip range, the nose range (5),6), and 7), respectively) and finally detected the characteristic points in a face(8)). As shown in table 1 and table 2, we compared proposed method with the previous method in terms of detection rate and authentication ratio for faces. The proposed method shows the fastest speed at the face search and the most accurate authentication ratio.

\section{Conclusions}

In this paper we utilized skin color detection to be able to extract the face region from color images of MCP, and settled the problems such as skin color definition and illumination, pointed out to the disadvantages included in the skin color detection. For the illumination problem, we established an alternative method using min-max normalization and histogram equalization for the illumination brightness, and used a statistical method with RGB values produced from two hundred data for the former problem.

\section{References}

1. Benjamin Miller, "Vital signs of identity", IEEE Spectrum pp,20-30, 1998.

2. D. Sanger, Y. Mlyake, "Algorithm for Face Extraction Based on Lip Detection," J. of Imaging Science and Technology, Vol.41, No.1, 1997.

3. B. Moghaddam, Was. Wahid, "Beyond Eigen Faces: Probabilistic Matching for Face Recognition," Prof. of IEEE ICAFGR'98, 1998.

4. T.Kandade, "Computer Recognition of Human faces," Birkhauser Verlag, 1997 